\title{
Distributed Test Capability Using Infrared Scene Projector Technology
}

David R. Anderson ${ }^{1}$, Ken Allred ${ }^{1}$, Kevin Dennen ${ }^{1}$, Patrick Roberts ${ }^{2}$, William R. Brown ${ }^{3}$, Ellis E. Burroughs ${ }^{3}$, Kenneth G. LeSueur ${ }^{3}$, and Tim Clardy ${ }^{3}$

\author{
1 ERC, Inc. \\ 555 Sparkman Drive, Executive Plaza, Suite 1622 \\ Huntsville, AL 35816 \\ \{danderson, kallred, kdennen\}@rttc.army.mil \\ 2 Sparta, Inc. \\ 4901 Corporate Dr NW \\ Huntsville, AL 35805 \\ \{proberts@rttc.army.mil\} \\ 3 US Army, Developmental Test Command \\ Redstone Technical Test Center \\ CSTE-DTC-RT-E-SA, Bldg. 4500 \\ Redstone Arsenal, AL 35898-8052 \\ \{rbrown, eburroughs, klesueur, tclardy\}@rttc.army.mil
}

\begin{abstract}
This paper describes a distributed test capability developed at the Redstone Technical Test Center (RTTC) in support of the Javelin Fire-and-Forget missile system. Javelin has a target acquisition sensor for surveying the battlefield, and a missile seeker staring focal plane array (FPA) for target tracking. Individually, these systems can be tested using scene projector capabilities at RTTC. But when combined, using this distributed capability, complete system acquisition to missile performance can be tested with the gunner in the loop. This capability was demonstrated at the Association of the United States Army (AUSA) conference in October of 2000. The Defense Research and Engineering Network (DREN) was used to connect the tactical target acquisition sensor in Washington, D.C. to the missile seeker and guidance section in Redstone Arsenal, in Huntsville, AL. The RTTC High Performance Computer provided scene projector control, distributed architecture support and real-time visible and infrared scene generation.
\end{abstract}

\section{Introduction}

This paper describes a distributed test capability developed by RTTC in Redstone Arsenal, AL, in support of the Javelin missile system. Javelin is a man portable, fire-and-forget antitank missile employed to defeat current and future threat armored combat vehicles. The Javelin consists of a missile in a disposable launch tube and a reusable Command Launch Unit (CLU) with a trigger mechanism and day/night sighting device for surveillance, and target acquisition and built-in test capabilities. The night sight is a long wave infrared (LWIR) scanning 
sensor. The missile locks on to the target before launch using an LWIR staring focal plane array and on-board processing, which also maintains target track and guides the missile to the target after launch. The capability to test this system in a distributed environment was demonstrated at the AUSA Annual Conference in Washington D.C. in October 2000. Two RTTC scene projector systems utilizing micro-resister array technology were used to support this effort. The missile was located at the RTTC Electro-Optical Subsystem Flight Evaluation Laboratory (EOSFEL) on Redstone Arsenal, AL. In Washington D.C. the Dynamic Infrared Scene Projector (DIRSP) Engineering Grade Array (DEGA) was used to project dynamic scenes into the CLU. Two real-time infrared scene generators were synchronized and running simultaneously at both locations. These scene generators along with the IR scene projectors allowed both sensors to be submerged into the same virtual environment. Targets moving on the database did so on both databases at the same time. The Defense Research and Engineering Network (DREN) was used to pass data between the two facilities.

\section{DIRSP Engineering Grade Array (DEGA)}

The DEGA infrared scene projector system uses resister array technology that was developed by Honeywell under the RTTC DIRSP program. The arrays are made up of 544 x 672 micro-emitter pixels (365,568 total pixels). Pixel pitch in the 544 direction is designed to be $45 \mathrm{um}$ and pixel pitch in the 672 direction is designed to be $61 \mathrm{um}$. This means that the physical size of the array format is 0.96 inches by 1.62 inches.

The resister array spectral characteristics accommodate the LWIR CLU target acquisition sensor of the Javelin system. The DEGA projects ground based scenes with an maximum apparent (effective blackbody) temperature of $373 \mathrm{~K}$, in the LWIR spectrum. The resister array is contained in a vacuum environment and mounted to a heat sink maintained at $238 \mathrm{~K}$. The micro-emitters are designed to ensure a temperature accuracy of $0.05 \mathrm{~K}$ and a design goal for DEGA is to have $0.03 \mathrm{~K}$ delta temperature accuracy and resolution.

The DEGA has a projection optics subsystem that contains optical elements necessary to project an image of the IR emitter array onto the entrance aperture of the sensor under test. Control electronics consist of all the processing and interface electronics as well as the software required to control the system. Finally, the computer image generator consists of the hardware and software necessary to render and display high resolution complex scene images in real-time at the required frame rates for testing.

\section{EOSFEL}

The EOSFEL is a state-of-the-art facility for testing EO missile guidance sections and control systems in a 6 degree-of-freedom (6-DOF), hardware-in-the-loop (HWIL) simulation environment. This closed-loop simulation capability expands 
current subsystem/component testing to include evaluation of system level performance under tactical flight scenarios. The EOSFEL provides a non-destructive environment for performing missile subsystem test throughout the weapon system life-cycle (i.e. design, development, qualification, production, and stockpile reliability).

The EOSFEL maintains state-of-the-art equipment for performing these various test/simulation exercises. A description of the major EOSFEL test/simulation equipment is presented in Table 1

Table 1. Major EOSFEL Equipment

\begin{tabular}{|c|c|}
\hline Simulation/Test Equipment & Description \\
\hline $\begin{array}{c}\text { Flight Motion Simulator (FMS) } \\
\text { Mountable Infrared Scene } \\
\text { Projector (FIRSP) }\end{array}$ & $\begin{array}{c}\text { FMS Mounted WISP 512x512 Wideband IR Resistor Array, } \\
\text { LWIR Optics, High Speed Drive Electronics, Real-Time Non- } \\
\text { Uniformity Correction (NUC), 2D Image Playback } \\
\text { Electronics, Vacuum \& Temperature Control }\end{array}$ \\
\hline $\begin{array}{c}\text { 3D Computer Image Generator } \\
\text { (CIG)/ Real-time Computer }\end{array}$ & $\begin{array}{c}\text { SGI Onyx2 InfiniteReality, 16 R12K Processors, 2 IR3 } \\
\text { Graphics Pipes, 6 Raster Managers w/ 256 Mb Texture, Real- } \\
\text { time Digital Video Port Interface to IRSP }\end{array}$ \\
\hline Real-Time I/O Linkage System & DACs, ADCs, Discrete I/O, Bit3 \& Scramnet Interfaces \\
\hline Flight Motion Simulator (FMS) & $\begin{array}{c}\text { Flight Motion Simulator (FMS) } \\
8 \text { inch dia. UUTs }\end{array}$ \\
\hline Dynamic Fin Loader (DFL) & Electro-Servo Driven 4 Channel Dynamic Loader, \\
& Up to 14 inch dia. CASs \\
\hline Environmental Conditioning & $\begin{array}{c}\text { Temperature Conditioning of Seeker, CAS, \& ESAF : -50 to } \\
+150^{\circ} \mathrm{F}\end{array}$ \\
\hline
\end{tabular}

\section{Distributed Test Configuration}

For this distributed exercise, two real-time computers were connected via a combination of an ATM Virtual Private Network supplied by the High Performance Computing Management Office (HPCMO) named DREN (Defense Research and Engineering Network) and commercial T1 leased lines for extending the connectivity to the metro Washington D.C. area. Figure 1 is a simple diagram that illustrates some of the components of the distributed test configuration. The real-time computer at the Redstone Technical Test Center operates as a distribution center for the HPCMO which provides extensive computer processing capabilities and high-bandwidth connections to remote sites. It was used to perform real-time scene generation, facility control, and network communications. The second real-time computer was transported to Washington D.C. along with the mobile DEGA scene projector and support electronics. A virtual prototype CLU was used to provide a gunner interface to control the Javelin system. A 
tactical CLU was mounted to stare into the DEGA scene projector. Using the gunner interface, the gunner had complete control of the missile system. The control switches are designed to slave the tactical CLU. Functions like FOV select, focus, and level and gain control on the gunner interface slave the tactical CLU. The gunner interface has laser ring gyros that monitor the motion of the gunner. Displacements in pitch and yaw are sensed and sent to the real-time computer. The real-time scene projected to the sensor under test, will move accordingly, based on the sensed motion of the gunner. When the gunner looks into the gunner interface eyepiece, he will see this motion.

There are several components that were modeled in the virtual world. A tank driving station was developed. It allowed a driver to use a joystick and drive around in the virtual scene. The tank position was continuously updated on both scene generators simultaneously. The station allowed the tank to be moved in any direction at any rate within the capabilities of the tank. As the tank moved around, the Javelin gunner, using the gunner interface, would engage the tank and fire the missile located in the EOSFEL. Tank position would update for the duration of the flight.

\subsection{Daysight Mode}

While in day mode, the gunner views the visible database. This scene is generated in real-time on the DEGA control computer. The gyros monitor gunner motion and send that information back to the real-time computer. The scene generator receives the displacement information and updates the look angles in the scene generator. At $30 \mathrm{~Hz}$, the gunner gets an updated view of the virtual world from his new perspective.

\subsection{CLU Nightsight}

During target acquisition, the gunner interface allows the operator to choose day sight or night. While in night mode, the gunner is looking at actual FLIR video from the tactical CLU looking into the DEGA scene projector. This is made possible by a Javelin test set that is interfaced to the tactical CLU. The test set grabs digital CLU video in real-time and sends the imagery to the gunner interface with very little latency. In this mode, the gunner will continue to have the ability to pan the virtual database in search of a potential target.

\subsection{Seeker Video}

The gunner will center the target in the FOV, and if ready to engage the target, will switch to seeker mode. When the gunner pulls the seeker mode trigger on the gunner interface, it sends a command back to the missile system in Redstone, and powers on the missile and starts the initialization. Initialization includes software download and cryogenic cooling of the focal plane array. Once the missile starts to image the scene, that video is sent back to the gunner interface over the 


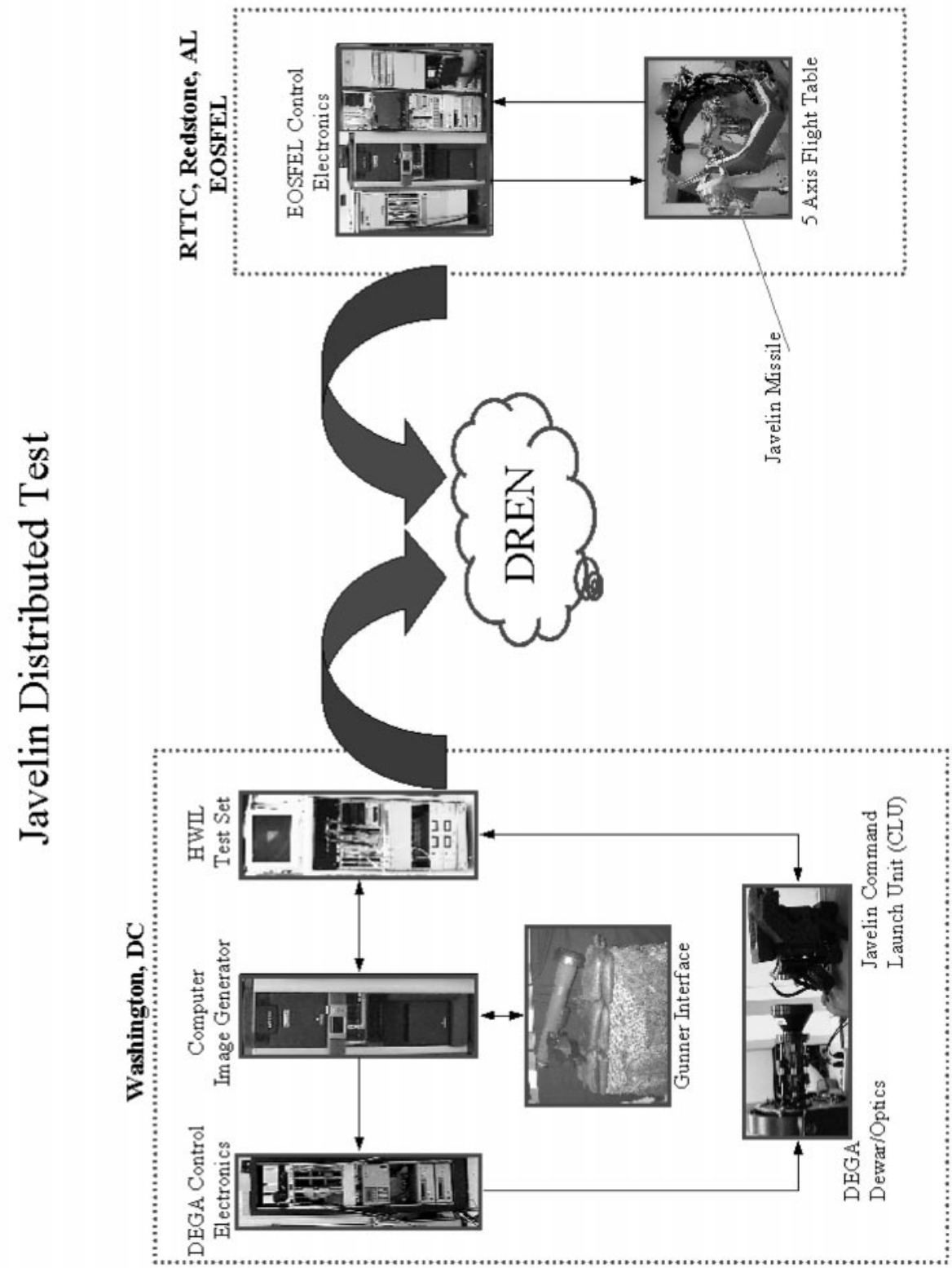

Fig. 1. Distributed Test Configuration 
DREN. At this time, the gunner in Washington D.C. is completely slaving that missile in Redstone. The operator uses the gunner interface to size track gates, lock-on the target, and pull the fire trigger. When the missile is launched, it will go through a complete hardware-in-the-loop (HWIL) firing in the EOSFEL.

\section{$5 \quad$ Network Infrastructure}

For the AUSA demo, two separate UDP sockets were established for communication. One UDP port was dedicated for reception of missile video and EOSFEL lab status data and the other UDP socket was dedicated to transmitting mission and scenario feedback. Approximately 5000 bytes were transmitted by the EOSFEL and 164 bytes were received. The timing of the data transfers were scheduled at $30 \mathrm{HZ}$ but an effective rate of approximately $28 \mathrm{HZ}$ was achieved during the demo. Several preliminary tests were conducted prior to the AUSA demo and the maximum latency was required to be less than 100 milliseconds in order to inject a smooth gunner motion into the HWIL missile track loop. Round trip latency from the EOSFEL at Redstone, to the AUSA demo was measured by a simple loop back test and was found to be approximately 20 milliseconds. The physical network connection was fairly extensive. The required data was transferred using a combination of the DREN and T1 networks. The EOSFEL ATM switch connected to an ATM switch in the RTTC HPC Distributed Center. From there, the ATM line runs to the CIC switch on Redstone. The signals then entered the DREN cloud. The Naval Research Lab in Washington, DC was the nearest DREN connection to the AUSA conference center. From that facility, the network goes through 2 leased T-1 lines and several routers to a switch that connects directly to the Onyx2 system controlling the DEGA scene projector.

\section{Scene Generation and Databases}

Scene Generation is the construction of radiometrically and visually calibrated digital images representing real-world situations that one would encounter on the test range or battlefield. These scenes may be based upon measured fieldtest imagery (empirical), physics-based 3-D computer models (predictive), or a combination of empirical and predictive sources. The scenes generated are then converted to a thermal or visual scene that is projected into the entrance aperture of a thermal imaging system.

Imagery that was projected into the CLU and the Javelin FPA was required to be generated in the Long Wave Infrared (LWIR) spectral bands. Injected day sight video is required to be in the visible spectrum. The LWIR imagery was projected into the tactical seeker and CLU from the FIRSP and the DEGA located at RTTC and Washington, D.C., respectively. The imagery from the tactical hardware was then acquired digitally and input into the viewing aperture of the gunner interface to give the gunner the appearance of tactical battlefield scenes. The visible spectrum was also generated and input digitally into the viewing aperture of the gunner interface. During the engagement exercise, the 
gunner could readily switch scenes between the visible, night sight, and seeker view. In order for the gunner not to perceive shifts in the presented imagery, the scenes presented to the gunner had to be correlated. In order to correlate the projected scenes, it was required that the visible and LWIR terrain and target databases be geometrically and geographically correlated. This was accomplished by placing the visible and LWIR textures on the same elevation map. Several databases have been correlated in the visible, LWIR and MWIR spectrums by RTTC under the scope of the DTC Virtual Proving Ground (VPG).

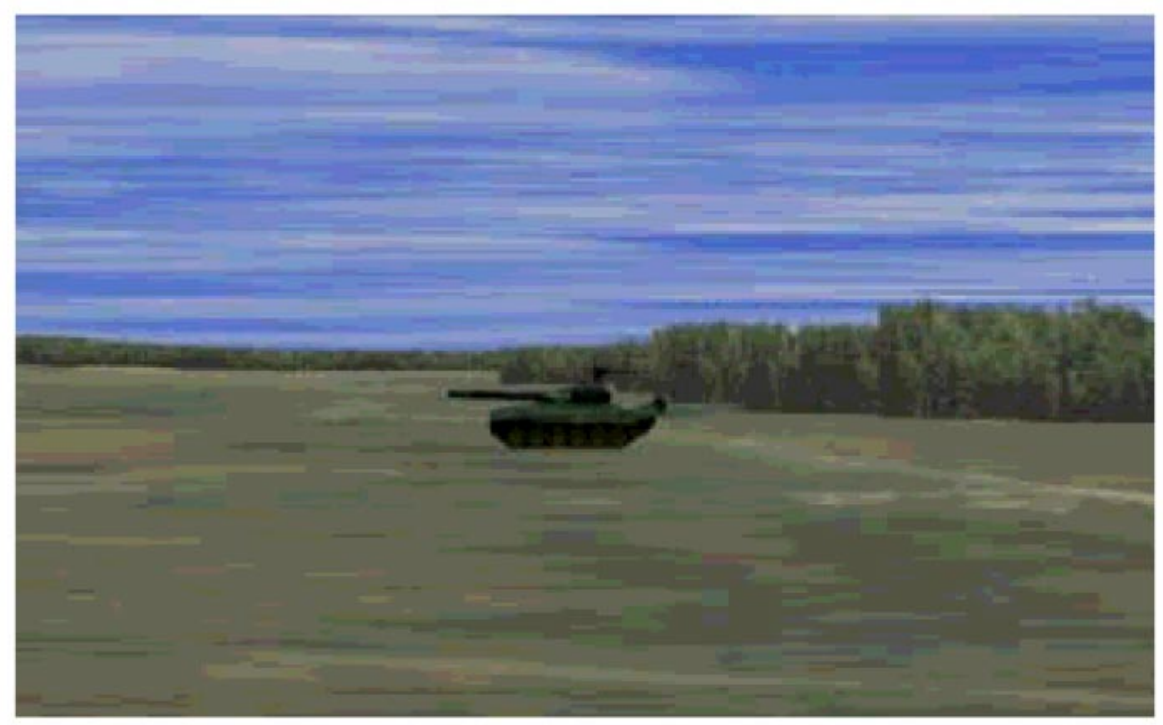

Fig. 2. TA-3 Visible Database and Target

\section{Conclusion}

Using multi-spectral scene generation and projection techniques provides earlier realistic testing which allows accelerated system design maturity and involves the user in early development. This testing technique provides a re-configurable cost effective supplement to destructive flight testing. The multi-spectral scene generation has also proven useful in combining Operational and Developmental test activities as well as rehearsals for live/field testing.

Another RTTC effort in the area of distributed multi-spectral scene generation is linking with weapon system developers. RTTC is the DoD sponsor in establishing a DREN connection at the Raytheon Javelin Lab in Tucson AZ. The connection provides the developer with real-time High Performance Computing (HPC) resources and the EOSFEL and EOTASEL HWIL testing labs 


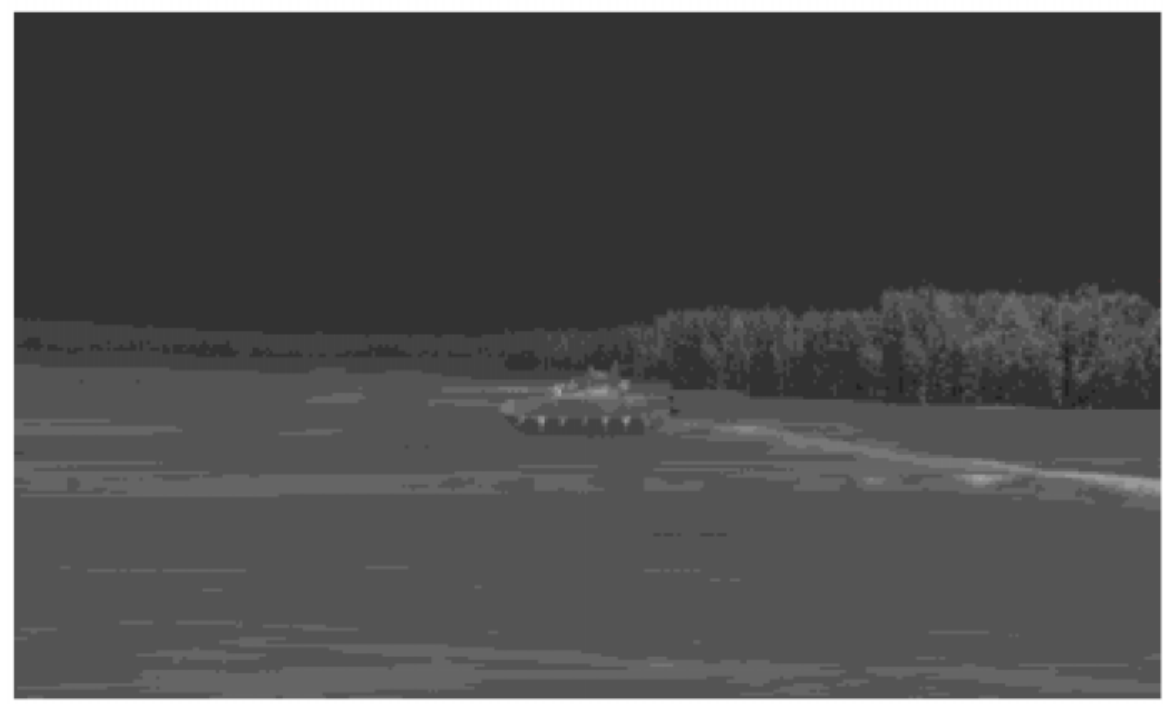

Fig. 3. TA-3 LWIR Database and Target

where multiple scene projectors are used for missile and target acquisition sensor testing.

The TOW Fire \& Forget program will be tested using multiple scene generators, projectors, and IR databases. The system will employ a LWIR Target Acquisition Sensor (TAS) and a mid wave IR missile seeker. Upon missile power up the system will perform an automatic boresight on the two sensors and hand-off target information from the TAS to the missile. In order to perform qualification and pre-flight testing RTTC will use EOSFEL and EOTASEL scene generators and projectors to provide real-time, simultaneous, spatially correlated, in-band IR scenes to both sensors in their respective wave bands. 